Physics

Physics Research Publications

MOJAVE: Monitoring of jets in AGN with VLBA experiments. IV. The parent luminosity function of radio-loud blazars

M. Cara M. L. Lister

This paper is posted at Purdue e-Pubs.

http://docs.lib.purdue.edu/physics_articles/642 
The Astrophysical Journal, 674:111-121, 2008 February 10

(C) 2008. The American Astronomical Society. All rights reserved. Printed in U.S.A.

\title{
MOJAVE: MONITORING OF JETS IN AGN WITH VLBA EXPERIMENTS. IV. THE PARENT LUMINOSITY FUNCTION OF RADIO-LOUD BLAZARS
}

\author{
M. CARA \\ Department of Physics, Purdue University, 525 Northwestern Avenue, West Lafayette, IN 47907; mcara@physics.purdue.edu \\ AND \\ M. L. LISTER \\ Department of Physics, Purdue University, 525 Northwestern Avenue, West Lafayette, IN 47907; mlister@physics.purdue.edu \\ Received 2007 February 15; accepted 2007 November 2
}

\begin{abstract}
We use a complete sample of active galactic nuclei (AGN) selected on the basis of relativistically beamed $15 \mathrm{GHz}$ radio flux density (MOJAVE: Monitoring of Jets in AGN with VLBA Experiments) to derive the parent radio luminosity function (RLF) of bright radio-selected blazar cores. We use a maximum likelihood method to fit a beamed RLF to the observed data and thereby recover the parameters of the intrinsic (unbeamed) RLF. We analyze two subsamples of the MOJAVE sample: the first contains only objects of known FR II class, with a total of 103 sources, and the second subsample adds 24 objects of uncertain FR class for a total of 127 sources. Both subsamples exclude four known FR I radio galaxies and two gigahertz-peaked spectrum sources. We obtain good fits to both subsamples using a single power law intrinsic RLF and a pure density evolution function of the form $z^{m} \exp \left\{-1 / 2\left[\left(z-z_{0}\right) / \sigma\right]^{2}\right\}$. We find that a previously reported break in the observed MOJAVE RLF actually arises from using incomplete bins (because of the luminosity cutoff) across a steep and strongly evolving RLF, and does not reflect a break in the intrinsic RLF. The derived space density of the parent population of the FR II sources from the MOJAVE sample (with $L_{15 \mathrm{GHz}} \geq 1.3 \times 10^{25} \mathrm{~W} \mathrm{~Hz}^{-1}$ ) is approximately $1.6 \times 10^{3} \mathrm{Gpc}^{-3}$.

Subject headings: BL Lacertae objects: general — galaxies: active — galaxies: evolution galaxies: luminosity function, mass function — quasars: general
\end{abstract}

\section{INTRODUCTION}

The radio luminosity function (RLF) of active galactic nuclei (AGN) and its redshift dependence are important quantities in understanding the physics of AGN and their cosmological evolution. In the case of AGN selected on the basis of relativistic emission (i.e., blazars), it can also provide information about the parent population from which an observed sample is drawn. A parameterized luminosity function (LF) can also be useful for producing Monte Carlo simulations of populations to compare with statistical properties of observed AGN (e.g., Lister \& Marscher 1997) as well as to study those properties of AGN that are difficult to observe directly. The intrinsic RLF can also be useful for predicting the number of $\gamma$-ray blazars to be observed by future surveys (e.g., GLAST; also, see Lister \& Marscher 1999) as well as for determining how rare individual blazars are in the general AGN population.

According to contemporary AGN unification schemes (see review by Urry \& Padovani 1995), various observed classes of AGN (e.g., radio galaxies, quasars, and BL Lac objects) can be the result of different orientations of essentially the same type of object. One can test unification schemes using statistical approaches. For example, if BL Lac objects are highly beamed versions of lower power radio galaxies, then the number of BL Lac objects should be much smaller then the number of parent radio galaxies, because BL Lac objects are oriented at a small angle to the line of sight. Previously, Urry et al. (1991), Padovani \& Urry (1992), and Urry \& Padovani (1995) applied relativistic beaming corrections (e.g., Cohen et al. 2007) to the RLF of high power radio galaxies and found it to be compatible with the observed RLF of a sample of flat-spectrum, radio-loud quasars. Jackson \& Wall (1999) proposed a dual-population unified scheme in which
(1) the high-power FR II radio galaxies are the parents of all radio quasars and some BL Lac-type objects, and (2) moderatepower FR I radio galaxies are the parents of the remaining BL Lac-type objects. They tested this model by beaming (using Monte Carlo jet populations with a single bulk Lorentz factor) the low-frequency radio data and comparing them with highfrequency radio data.

The MOJAVE AGN sample (Lister \& Homan 2005) is the first large, radio-selected AGN sample for which jet kinematic and apparent superluminal speed information are available (Kellermann et al. 2004; M. L. Lister et al. 2008, in preparation). It is complete with respect to relativistically beamed jet emission, and therefore provides a unique opportunity to learn about the intrinsic (parent) RLF of blazars. The determination of the intrinsic (nonbeamed) RLF is complicated, however, by relativistic beaming and selection effects. The radio emission from an AGN is highly enhanced by Doppler boosting if its jet is relativistic and aligned close to the line of sight. A flux density-limited sample of AGN will therefore contain not only sources with high intrinsic luminosity, but also sources with lower intrinsic luminosities whose flux densities are Doppler boosted because of their orientation. The effect of Doppler beaming on the observed RLF was first calculated for single Lorentz factors (Urry \& Shafer 1984) and later extended for distributions of Lorentz factors (Urry \& Padovani 1991). Lister (2003) extended these studies by deriving fully analytical expressions for the Doppler factor distributions and beamed RLFs.

Previous studies (see, e.g., Padovani \& Urry 1992) started with the assumption that the intrinsic parent LF was that of the FR II galaxies, and then applied beaming in order to compare it with the LFs of the steep-spectrum radio quasars (SSRQ) and flat-spectrum radio quasars (FSRQ). Since their sample was 
selected at a much lower radio frequency $(2 \mathrm{GHz})$, it was subject to contamination by sources with large extended emission, as well as uncertainties in the classification of the sources into SSRQ and FSRQ based on a somewhat arbitrary spectral index cutoff of $\alpha_{\text {rad }}=-0.5$. In addition, in order to simulate the beamed LFs of SSRQ and FSRQ it was necessary to know the ratio of the core to extended emission. Padovani \& Urry (1992) assumed a linear relationship between the beamed and unbeamed luminosities, but found that they needed different factors to produce good fits to the SSRQ and FSRQ. A somewhat similar approach was used by Jackson \& Wall (1999) in determining the beaming models of the parent populations in their dual-population unification scheme.

The MOJAVE sample is different from previous samples in that it is selected on the basis of (highly variable) radio flux densities at a high frequency of $15 \mathrm{GHz}$, thus effectively eliminating contamination from extended source emission. The uncertainties surrounding the spectral index cutoff and core-to-extended emission ratios are likewise alleviated.

In this paper we use the maximum likelihood method to fit a beamed RLF to the observed data, from which we recover the RLF parameters of the parent population of the MOJAVE sample. These parameters will be used in upcoming studies of the effects of beaming on the blazar properties derived from flux limited samples.

The outline of the paper is as follows: In $\S 2$ we describe the observational sample and our method for dealing with incomplete redshift information. In $\S 3.1$ we describe our parameterization of the RLF, and in $\S 3.2$ we describe the method used to find the optimized model parameters and constraints on the fits. We present the results of the model fitting in $\S 4$ and summarize our findings in $\S 5$.

Throughout this paper we assume (unless stated otherwise) a cosmology with $\Omega_{m}=0.3, \Omega_{\Lambda}=0.7, \Omega_{r}=0$, and $H_{0}=$ $70 \mathrm{~km} \mathrm{~s}^{-1} \mathrm{Mpc}^{-1}$. All luminosities are quoted as monochromatic luminosities at specific frequency $\nu$. We also adopt the following convention for the spectral index, $\alpha_{\mathrm{rad}}: S_{\nu} \propto \nu^{\alpha_{\mathrm{rad}}}$.

\section{OBSERVATIONAL DATA}

\subsection{Sample Description}

The MOJAVE AGN sample (Lister \& Homan 2005) consists of all 133 known bright AGN with galactic latitude $|b|>2.5^{\circ}$, $\mathrm{J} 2000.0$ declination greater than $-20^{\circ}$, and compact $\left(\mathrm{VLBA}^{1}\right.$ ) flux density exceeding $1.5 \mathrm{Jy}$ at $15 \mathrm{GHz}$ (2 Jy for sources with $\delta<0$ ) at any epoch between 1994 January 1 and 2003 December 31. The sky area covered by the MOJAVE survey is $6.00912 \mathrm{sr}$ for the northern sky and $2.08012 \mathrm{sr}$ for the southern sky. The sample is selected on the basis of beamed jet emission only. The contribution from the large-scale radio emission is effectively excluded by using the milliarcsecond scale (VLBA) $15 \mathrm{GHz}$ flux density, since the former tends to be diffuse and has a steep radio spectrum.

Many of the MOJAVE sources exhibit high flux density variability and have been selected based on their largest flux density values, thus potentially creating a source of selection bias. However, in a detailed study of AGN variability, Lister (2001) concluded that the effect of the variability on the sample selection is small in moderately sized samples because the majority of highly beamed sources in the parent population (which are preferentially selected in beamed emission-selected samples; see, e.g.,

\footnotetext{
1 The Very Long Baseline Array is operated by the National Radio Astronomy Observatory, which is a facility of the National Science Foundation operated under cooperative agreement by Associated Universities, Inc.
}

Vermeulen \& Cohen 1994) would lie well above the survey flux limit and will be selected regardless of their flaring levels. Those sources just above the survey limit would be statistically balanced by other sources lying just below.

We present basic data for the MOJAVE sample, including Fanaroff-Riley (FR) and optical classifications for the MOJAVE sample in Table 1. For optical class we used the Veron-Cetty \& Veron (2006) catalog, as well as the NASA/IPAC Extragalactic Database (NED). We note that the optical classification of blazars into BL Lac objects and optically violently variable (OVV) quasars remains controversial (see, e.g., Antonucci 1993; Kovalev et al. 2005), but this is not essential to our analysis, which is based on Fanaroff-Riley classes. We base our Fanaroff-Riley classification of the MOJAVE sources on their extended radio morphology. We assign an uncertain FR class to BL Lac objects and intermediate BL Lac/HPQ sources for which morphological FR classification is difficult (i.e., core, halo, unusual morphologies or otherwise lack of prominent hot spots). For quasars only, in situations were morphological classification is difficult we rely on the source's luminosity to assign it a FR class. At $178 \mathrm{MHz}$ the Fanaroff-Riley divide occurs at about $L_{178 \mathrm{MHz}} \approx 10^{25.3} \mathrm{~W} \mathrm{~Hz}^{-1}$, but this transition depends on the host galaxy magnitude (see Owen \& Ledlow 1994; Ledlow \& Owen 1996) and sources close to this luminosity can be of either class. The line separating the two classes at $1.4 \mathrm{GHz}$ in Figure 1 of Ledlow \& Owen (1996) can be approximated as $\log L_{\mathrm{FR} \text { break }} \approx 10.2-(2 / 3) M_{R}^{\text {host }}$ over the range $-25<M_{R}^{\text {host }}<-21$ (assuming their cosmology of $q_{0}=0$ and $\left.H_{0}=75 \mathrm{~km} \mathrm{~s}^{-1} \mathrm{Mpc}^{-1}\right)$. We used this equation to find the FR break luminosity, $L_{\mathrm{FR} \text { break }}$, for a given host magnitude. We considered any quasar with an inconclusive morphology to be of FR II class if its luminosity at $1.4 \mathrm{GHz}$ is at least an order

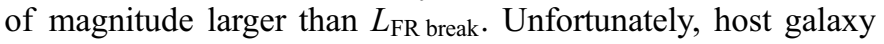
magnitudes are available only for six of the 91 of the quasars in our sample. Pagani et al. (2003) found that the average absolute magnitude of the host galaxies radio loud quasars at low redshifts $(z<0.5)$ is $\left\langle M_{R}\right\rangle=-24.0 \pm 0.5, q_{0}=0$, and $H_{0}=$ $50 \mathrm{~km} \mathrm{~s}^{-1} \mathrm{Mpc}^{-1}$, which when converted to the cosmology of Ledlow \& Owen (1996) becomes $\left\langle M_{R}\right\rangle \approx-23.1$. We assume $M_{R}=-23.1$ for the 85 quasars for which we could not find information in the literature on the absolute magnitudes of their host galaxies. We have computed the luminosities at $1.4 \mathrm{GHz}$ from the fluxes found on NED (we used the largest listed flux) assuming a spectral index $\alpha_{\text {rad }}=-0.7$. All of the quasars with uncertain morphological FR classifications ended up as FR II class according to this model. Of the eight galaxies in the MOJAVE sample, three galaxies $(0007+106$ : III $\mathrm{Zw} 2,0415+379$ : 3C 111, and 1957+405: Cygnus A) show FR II morphologies, another four galaxies (0238-084: NGC 1052, 0316+413: 3C 84, 0430+052: 3C 120, and 1228+126: M87) show FR I morphologies, and the galaxy $2021+614: \mathrm{OW}+637$ is a gigahertz-peaked spectrum (GPS) source. The other GPS source in the MOJAVE sample is the quasar $0742+103$. Redshifts are available from NED for all but 12 sources (6 optically featureless BL Lac objects, and 6 sources without optical identifications).

According to a contemporary unification scheme (e.g., Urry \& Padovani 1995), the parent population of BL Lac objects is identified with FR I type galaxies. However, the issue of parent populations for BL Lac objects remains under debate. Recent studies of the host galaxy and extended radio emission of radioselected, low-energy peaked BL Lac objects (e.g., Cassaro et al. 1999; Rector \& Stocke 2001; Kotilainen et al. 2005) appear to rule out the FR I-BL Lac unification scheme in its simplest form.

Jackson \& Wall (1999) proposed a dual-population scheme in which FR II radio galaxies are the misaligned parents of 
TABLE 1

The MOJAVE SAmple

\begin{tabular}{ccccc}
\hline \hline & \multicolumn{5}{c}{$S_{15}$} \\
B1950 Name & $z$ & $($ Jy) & FR Class & Optical Class \\
$(1)$ & $(2)$ & $(3)$ & $(4)$ & $(5)$ \\
\hline
\end{tabular}

MOJAVE sources of FR II class

\begin{tabular}{|c|c|c|c|c|}
\hline $0007+106 \ldots \ldots \ldots \ldots \ldots \ldots$ & 0.0893 & 2.300 & 2 & G \\
\hline $0016+731 \ldots \ldots \ldots \ldots \ldots \ldots$ & 1.781 & 2.260 & $2^{\mathrm{a}}$ & $\mathrm{Q}$ \\
\hline 0048-097 ................... & $\ldots$ & 2.160 & 2 & $\mathrm{BL}$ \\
\hline $0059+581 \ldots \ldots \ldots \ldots \ldots \ldots$ & 0.643 & 3.307 & $2^{a}$ & $\mathrm{Q}$ \\
\hline $0106+013 \ldots \ldots \ldots \ldots \ldots \ldots$ & 2.107 & 2.972 & 2 & Q \\
\hline $0133+476 \ldots \ldots \ldots \ldots \ldots \ldots$ & 0.859 & 4.953 & $2^{\mathrm{a}}$ & Q \\
\hline $0202+149 \ldots \ldots \ldots \ldots \ldots \ldots$ & 0.405 & 2.293 & $2^{\mathrm{a}}$ & Q \\
\hline $0202+319 \ldots \ldots \ldots \ldots \ldots \ldots$ & 1.466 & 2.284 & 2 & Q \\
\hline 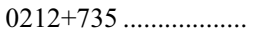 & 2.367 & 2.842 & $2^{a}$ & $\mathrm{Q}$ \\
\hline $0215+015 \ldots \ldots \ldots \ldots \ldots \ldots$ & 1.715 & 1.532 & 2 & $\mathrm{BL} / \mathrm{HPQ}$ \\
\hline 0224+671 .................. & 0.523 & 2.450 & 2 & Q \\
\hline 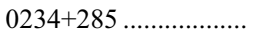 & 1.207 & 4.045 & 2 & $\mathrm{Q}$ \\
\hline $0235+164 \ldots \ldots \ldots \ldots \ldots \ldots$ & 0.940 & 1.731 & 2 & $\mathrm{BL} / \mathrm{HPQ}$ \\
\hline $0333+321 \ldots \ldots \ldots \ldots \ldots \ldots$ & 1.263 & 2.249 & 2 & Q \\
\hline 0336-019 .................. & 0.852 & 3.452 & 2 & Q \\
\hline 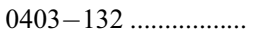 & 0.571 & 2.750 & $2^{\mathrm{a}}$ & Q \\
\hline $0415+379 \ldots \ldots \ldots \ldots \ldots \ldots$ & 0.0491 & 5.976 & 2 & G \\
\hline $0420-014$.................. & 0.915 & 10.438 & 2 & Q \\
\hline 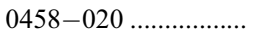 & 2.291 & 2.325 & $2^{a}$ & Q \\
\hline $0528+134 \ldots \ldots \ldots \ldots \ldots \ldots$ & 2.070 & 7.945 & $2^{\mathrm{a}}$ & Q \\
\hline $0529+075 \ldots \ldots \ldots \ldots \ldots . . . . . . . .$. & 1.254 & 1.630 & 2 & Q \\
\hline $0529+483 \ldots \ldots \ldots \ldots \ldots \ldots$ & 1.162 & 1.662 & 2 & Q \\
\hline $0552+398 \ldots \ldots \ldots \ldots \ldots \ldots$ & 2.363 & 5.020 & $2^{\mathrm{a}}$ & Q \\
\hline $0605-085$................... & 0.872 & 2.797 & 2 & Q \\
\hline 0607-157 ................... & 0.324 & 7.263 & $2^{\mathrm{a}}$ & Q \\
\hline $0642+449 \ldots \ldots \ldots \ldots \ldots \ldots$ & 3.408 & 4.310 & $2^{\mathrm{a}}$ & Q \\
\hline $0727-115 \ldots \ldots \ldots \ldots \ldots$ & 1.591 & 5.125 & $2^{\mathrm{b}}$ & Q \\
\hline 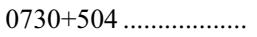 & 0.720 & 1.440 & 2 & Q \\
\hline 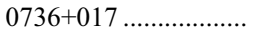 & 0.191 & 2.649 & 2 & Q \\
\hline $0738+313 \ldots \ldots \ldots \ldots \ldots . . . . . . .$. & 0.630 & 2.868 & 2 & Q \\
\hline $0748+126 \ldots \ldots \ldots \ldots \ldots \ldots$ & 0.889 & 3.248 & 2 & Q \\
\hline 0804+499 .................. & 1.432 & 2.380 & 2 & Q \\
\hline 0805-077 ................. & 1.837 & 3.488 & $2^{\mathrm{b}}$ & $\mathrm{Q}$ \\
\hline $0814+425 \ldots \ldots \ldots \ldots \ldots \ldots$ & 0.245 & 1.810 & 2 & $\mathrm{BL} / \mathrm{HPQ}$ \\
\hline $0827+243 \ldots \ldots \ldots \ldots \ldots \ldots$ & 0.941 & 1.989 & 2 & Q \\
\hline $0836+710 \ldots \ldots \ldots \ldots \ldots \ldots$ & 2.218 & 2.237 & 2 & Q \\
\hline 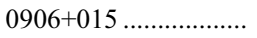 & 1.018 & 2.735 & 2 & Q \\
\hline $0917+624 \ldots \ldots \ldots \ldots \ldots \ldots$ & 1.446 & 1.970 & $2^{a}$ & Q \\
\hline $0923+392 \ldots \ldots \ldots \ldots \ldots \ldots$ & 0.698 & 12.683 & 2 & Q \\
\hline 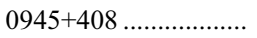 & 1.252 & 1.589 & 2 & Q \\
\hline $0955+476 \ldots \ldots \ldots \ldots \ldots \ldots$ & 1.873 & 1.715 & 2 & Q \\
\hline $1038+064 \ldots \ldots \ldots \ldots \ldots \ldots$ & 1.265 & 1.846 & $2^{\mathrm{a}}$ & Q \\
\hline $1045-188 \ldots \ldots \ldots \ldots \ldots$ & 0.595 & 2.339 & $2^{\mathrm{b}}$ & Q \\
\hline $1055+018 \ldots \ldots \ldots \ldots \ldots \ldots$ & 0.888 & 5.296 & 2 & $\mathrm{BL} / \mathrm{HPQ}$ \\
\hline $1124-186 \ldots \ldots \ldots \ldots \ldots$ & 1.048 & 2.819 & $2^{\mathrm{b}}$ & Q \\
\hline $1127-145 \ldots \ldots \ldots \ldots \ldots \ldots$ & 1.187 & 3.388 & 2 & Q \\
\hline $1150+812 \ldots \ldots \ldots \ldots \ldots \ldots$ & 1.250 & 1.651 & 2 & $\mathrm{Q}$ \\
\hline $1156+295 \ldots \ldots \ldots \ldots \ldots \ldots$ & 0.729 & 3.302 & $2^{\mathrm{a}}$ & $\mathrm{Q}$ \\
\hline $1219+044 \ldots \ldots \ldots \ldots \ldots \ldots$ & 0.965 & 1.678 & $2^{\mathrm{b}}$ & $\mathrm{Q}$ \\
\hline $1222+216 \ldots \ldots \ldots \ldots \ldots \ldots$ & 0.435 & 1.795 & 2 & $\mathrm{Q}$ \\
\hline $1226+023 \ldots \ldots \ldots \ldots \ldots \ldots$ & 0.158 & 41.399 & 2 & $\mathrm{Q}$ \\
\hline $1253-055 \ldots \ldots \ldots \ldots \ldots \ldots$ & 0.538 & 24.887 & 2 & Q \\
\hline $1308+326 \ldots \ldots \ldots \ldots \ldots \ldots$ & 0.997 & 3.982 & 2 & $\mathrm{BL} / \mathrm{HPQ}$ \\
\hline $1324+224 \ldots \ldots \ldots \ldots \ldots$ & 1.400 & 1.953 & $2^{\mathrm{a}}$ & Q \\
\hline $1334-127$................. & 0.539 & 8.868 & $2^{\mathrm{a}}$ & $\mathrm{Q}$ \\
\hline $1417+385 \ldots \ldots \ldots \ldots \ldots \ldots$ & 1.832 & 1.772 & $2^{a}$ & Q \\
\hline $1458+718 \ldots \ldots \ldots \ldots \ldots \ldots$ & 0.904 & 2.740 & 2 & Q \\
\hline $1502+106 \ldots \ldots \ldots \ldots \ldots \ldots$ & 1.839 & 1.956 & 2 & $\mathrm{Q}$ \\
\hline $1504-166 \ldots \ldots \ldots \ldots \ldots . . . . . . .$. & 0.876 & 2.031 & $2^{\mathrm{b}}$ & $\mathrm{Q}$ \\
\hline $1510-089$................... & 0.360 & 2.939 & 2 & Q \\
\hline 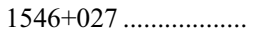 & 0.412 & 2.833 & 2 & $\mathrm{Q}$ \\
\hline
\end{tabular}

TABLE 1 -Continued

\begin{tabular}{|c|c|c|c|c|}
\hline $\begin{array}{c}\text { B1950 Name } \\
\text { (1) }\end{array}$ & $\begin{array}{c}z \\
(2)\end{array}$ & $\begin{array}{l}S_{15} \\
\text { (Jy) } \\
\text { (3) }\end{array}$ & $\begin{array}{c}\text { FR Class } \\
\text { (4) }\end{array}$ & $\begin{array}{c}\text { Optical Class } \\
\text { (5) }\end{array}$ \\
\hline \multicolumn{5}{|c|}{ MOJAVE sources of FR II class } \\
\hline $1548+056 \ldots \ldots \ldots \ldots \ldots \ldots$ & 1.422 & 2.917 & $2^{\mathrm{a}}$ & Q \\
\hline $1606+106 \ldots \ldots \ldots \ldots \ldots \ldots$ & 1.226 & 2.306 & 2 & Q \\
\hline $1611+343 \ldots \ldots \ldots \ldots \ldots \ldots$ & 1.401 & 5.672 & 2 & Q \\
\hline $1633+382 \ldots \ldots \ldots \ldots \ldots . . . . . .$. & 1.807 & 4.289 & 2 & Q \\
\hline 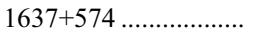 & 0.751 & 1.875 & $2^{\mathrm{a}}$ & Q \\
\hline $1638+398 \ldots \ldots \ldots \ldots \ldots \ldots$ & 1.666 & 1.608 & 2 & Q \\
\hline $1641+399 \ldots \ldots \ldots \ldots \ldots \ldots$ & 0.594 & 8.730 & 2 & Q \\
\hline 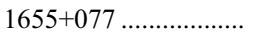 & 0.621 & 2.091 & 2 & Q \\
\hline $1726+455 \ldots \ldots \ldots \ldots \ldots \ldots$ & 0.714 & 2.184 & 2 & Q \\
\hline $1730-130 \ldots \ldots \ldots \ldots \ldots . . . . . .$. & 0.902 & 10.967 & 2 & Q \\
\hline $1739+522 \ldots \ldots \ldots \ldots \ldots \ldots$ & 1.379 & 1.766 & 2 & Q \\
\hline 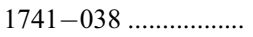 & 1.057 & 7.012 & $2^{\mathrm{a}}$ & Q \\
\hline $1758+388 \ldots \ldots \ldots \ldots \ldots$ & 2.092 & 1.745 & $2^{\mathrm{a}}$ & Q \\
\hline $1800+440 \ldots \ldots \ldots \ldots \ldots \ldots$ & 0.663 & 1.476 & 2 & $\mathrm{Q}$ \\
\hline $1803+784 \ldots \ldots \ldots \ldots \ldots \ldots$ & 0.680 & 2.543 & 2 & $\mathrm{BL} / \mathrm{HPQ}$ \\
\hline $1823+568 \ldots \ldots \ldots \ldots \ldots \ldots$ & 0.664 & 2.309 & 2 & $\mathrm{BL} / \mathrm{HPQ}$ \\
\hline 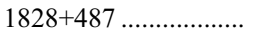 & 0.692 & 2.010 & 2 & Q \\
\hline $1849+670 \ldots \ldots \ldots \ldots \ldots \ldots$ & 0.657 & 1.708 & 2 & Q \\
\hline 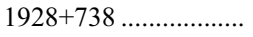 & 0.303 & 3.833 & 2 & Q \\
\hline $1936-155 \ldots \ldots \ldots \ldots \ldots . . . . . .$. & 1.657 & 2.439 & $2^{a}$ & Q \\
\hline 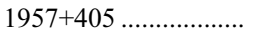 & 0.0561 & 1.680 & 2 & G \\
\hline 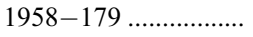 & 0.652 & 2.670 & $2^{\mathrm{a}}$ & Q \\
\hline $2005+403 \ldots \ldots \ldots \ldots \ldots \ldots$ & 1.736 & 2.767 & $2^{\mathrm{b}}$ & Q \\
\hline $2008-159$................... & 1.180 & 2.134 & $2^{\mathrm{b}}$ & Q \\
\hline $2037+511 \ldots \ldots \ldots \ldots \ldots \ldots$ & 1.687 & 2.337 & 2 & Q \\
\hline 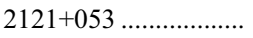 & 1.941 & 3.744 & $2^{\mathrm{a}}$ & Q \\
\hline $2128-123 \ldots \ldots \ldots \ldots \ldots \ldots . .$. & 0.501 & 3.182 & $2^{\mathrm{b}}$ & Q \\
\hline $2131-021 \ldots \ldots \ldots \ldots \ldots . .$. & 1.285 & 2.439 & $2^{\mathrm{a}}$ & $\mathrm{BL} / \mathrm{HPQ}$ \\
\hline 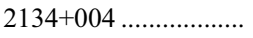 & 1.932 & 6.336 & 2 & Q \\
\hline $2136+141 \ldots \ldots \ldots \ldots \ldots \ldots$ & 2.427 & 2.75 & $2^{\mathrm{b}}$ & Q \\
\hline $2145+067 \ldots \ldots \ldots \ldots \ldots$ & 0.999 & 10.372 & $2^{\mathrm{a}}$ & Q \\
\hline $2201+171 \ldots \ldots \ldots \ldots \ldots \ldots . .$. & 1.076 & 1.986 & 2 & Q \\
\hline $2201+315 \ldots \ldots \ldots \ldots \ldots \ldots$ & 0.298 & 3.27757 & 2 & Q \\
\hline $2209+236 \ldots \ldots \ldots \ldots \ldots$ & 1.125 & 1.620 & $2^{a}$ & Q \\
\hline $2216-038 \ldots \ldots \ldots \ldots \ldots . . . . . . .$. & 0.901 & 2.536 & 2 & Q \\
\hline $2227-088 \ldots \ldots \ldots \ldots \ldots . . . . . .$. & 1.562 & 2.150 & 2 & Q \\
\hline $2230+114 \ldots \ldots \ldots \ldots \ldots \ldots$ & 1.037 & 4.855 & $2^{a}$ & Q \\
\hline $2243-123 \ldots \ldots \ldots \ldots \ldots \ldots$ & 0.630 & 2.559 & $2^{\mathrm{a}}$ & Q \\
\hline $2251+158 \ldots \ldots \ldots \ldots \ldots \ldots$ & 0.859 & 12.084 & 2 & Q \\
\hline 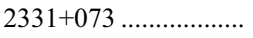 & 0.401 & 1.552 & 2 & Q \\
\hline $2345-167 \ldots \ldots \ldots \ldots \ldots \ldots$ & 0.576 & 2.536 & $2^{a}$ & Q \\
\hline $2351+456 \ldots \ldots \ldots \ldots \ldots \ldots$ & 1.986 & 1.814 & $2^{a}$ & $\mathrm{Q}$ \\
\hline
\end{tabular}

MOJAVE sources of uncertain FR class

\begin{tabular}{|c|c|c|c|c|}
\hline $0003-066 \ldots \ldots \ldots \ldots \ldots . . . . . . . .$. & 0.347 & 3.302 & $\ldots$ & BL \\
\hline 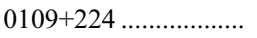 & $\ldots$ & 1.654 & $\ldots$ & BL \\
\hline $0119+115 \ldots \ldots \ldots \ldots \ldots \ldots$ & 0.570 & 2.007 & $\ldots$ & $\mathrm{BL} / \mathrm{HPQ}$ \\
\hline $0300+470 \ldots \ldots \ldots \ldots \ldots \ldots$ & $\ldots$ & 1.770 & $\ldots$ & BL \\
\hline 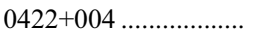 & $\ldots$ & 1.739 & $\ldots$ & BL \\
\hline $0446+112 \ldots \ldots \ldots \ldots \ldots \ldots$ & $\ldots$ & 2.256 & $\ldots$ & $\mathrm{U}$ \\
\hline 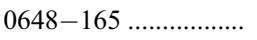 & $\ldots$ & 3.437 & $\ldots$ & $\mathrm{U}$ \\
\hline 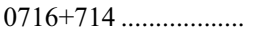 & $>0.52$ & 2.586 & $\ldots$ & BL \\
\hline $0735+178 \ldots \ldots \ldots \ldots \ldots \ldots$ & $>0.424$ & 1.635 & $\ldots$ & BL \\
\hline $0754+100 \ldots \ldots \ldots \ldots \ldots \ldots$ & 0.266 & 1.833 & $\ldots$ & $\mathrm{BL} / \mathrm{HPQ}$ \\
\hline 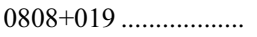 & 1.148 & 1.590 & $\ldots$ & BL \\
\hline $0823+033 \ldots \ldots \ldots \ldots \ldots \ldots$ & 0.506 & 2.467 & $\ldots$ & $\mathrm{BL} / \mathrm{HPQ}$ \\
\hline $0829+046 \ldots \ldots \ldots \ldots \ldots \ldots . .$. & 0.180 & 1.720 & $\ldots$ & BL \\
\hline $0851+202 \ldots \ldots \ldots \ldots \ldots \ldots$ & 0.306 & 4.375 & $\ldots$ & $\mathrm{BL} / \mathrm{HPQ}$ \\
\hline 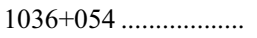 & $\ldots$ & 2.664 & $\ldots$ & $\mathrm{U}$ \\
\hline $1213-172 \ldots \ldots \ldots \ldots \ldots . .$. & $\ldots$ & 2.564 & $\ldots$ & $\mathrm{U}$ \\
\hline $1413+135$................... & 0.247 & 1.719 & $\ldots$ & $\mathrm{BL}$ \\
\hline $1538+149 \ldots \ldots \ldots \ldots \ldots \ldots$ & 0.605 & 1.630 & $\ldots$ & $\mathrm{BL} / \mathrm{HPQ}$ \\
\hline
\end{tabular}


TABLE 1 - Continued

\begin{tabular}{|c|c|c|c|c|}
\hline $\begin{array}{l}\text { B1950 Name } \\
\text { (1) }\end{array}$ & $\begin{array}{c}z \\
(2)\end{array}$ & $\begin{array}{l}S_{15} \\
\text { (Jy) } \\
\text { (3) }\end{array}$ & $\begin{array}{c}\text { FR Class } \\
\text { (4) }\end{array}$ & $\begin{array}{c}\text { Optical Class } \\
\text { (5) }\end{array}$ \\
\hline \multicolumn{5}{|c|}{ MOJAVE sources of uncertain FR class } \\
\hline 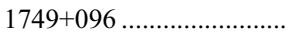 & 0.320 & 6.020 & $\ldots$ & $\mathrm{BL} / \mathrm{HPQ}$ \\
\hline $1751+288 \ldots \ldots \ldots \ldots \ldots \ldots \ldots . .$. & $\ldots$ & 2.015 & $\ldots$ & $\mathrm{U}$ \\
\hline $2021+317 \ldots \ldots \ldots \ldots \ldots \ldots \ldots . .$. & $\ldots$ & 2.158 & $\ldots$ & U \\
\hline 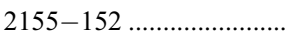 & 0.672 & 2.147 & $\ldots$ & $\mathrm{BL} / \mathrm{HPQ}$ \\
\hline 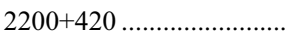 & 0.0686 & 5.669 & $\ldots$ & $\mathrm{BL} / \mathrm{HPQ}$ \\
\hline $2223-052 \ldots \ldots \ldots \ldots \ldots \ldots \ldots \ldots$ & 1.404 & 6.572 & $\cdots$ & $\mathrm{BL} / \mathrm{HPQ}$ \\
\hline \multicolumn{5}{|c|}{ Excluded MOJAVE sources } \\
\hline $0238-084$ & 0.0049 & 2.481 & 1 & $\mathrm{G}$ \\
\hline 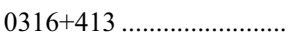 & 0.01756 & 12.908 & 1 & G \\
\hline $0430+052$ & 0.033 & 4.412 & 1 & $\mathrm{G}$ \\
\hline 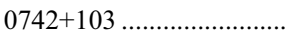 & 2.624 & 1.504 & $\ldots$ & Q \\
\hline 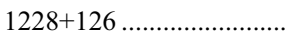 & 0.00436 & 2.969 & 1 & G \\
\hline 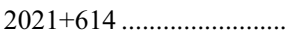 & 0.227 & 2.735 & $\ldots$ & G \\
\hline
\end{tabular}

Notes.-Col. (1): Source B1950 name. Col. (2): Redshift. Col. (3): Flux density at $15 \mathrm{GHz}$ in Jy. Col. (4): Fanaroff-Riley class. Col. (5): Optical class: BL = $\mathrm{BL}$ Lac, $\mathrm{Q}=$ quasar, $\mathrm{G}=$ radio galaxy, $\mathrm{BL} / \mathrm{HPQ}=\mathrm{BL} \mathrm{Lac} / \mathrm{High}$ Polarization Quasar (e.g., Veron-Cetty \& Veron 2000), U = unidentified.

${ }^{\text {a }}$ Classification based on luminosity at $1.4 \mathrm{GHz}$ when the morphology was inconclusive.

${ }^{\mathrm{b}}$ Classification based on luminosity at $1.4 \mathrm{GHz}$ when a kpc-scale image was unavailable.

flat-spectrum quasars and some BL Lac objects. We adopt this unification model and exclude four FR I galaxies (0238-084, $0316+413,0430+052$, and $1228+126)$ from the sample because they may belong to a different parent population and exhibit a different evolution from the rest of the sources. We also exclude the two GPS sources $0742+103$ and $2021+614$. In our analysis we will use two samples: one containing known FR II sources (hereafter, the "known FR II sample") and a second sample comprising both known FR II and uncertain FR class sources (hereafter, the "full sample"). The known FR II sample contains 103 sources (91 quasars, 3 FR II galaxies, 1 BL Lac, and 8 sources of the intermediate BL Lac/High Polarization Quasar [HPQ] class; e.g., Veron-Cetty \& Veron 2000). One of the sources in this sample lacks redshift information. The full sample contains 127 sources (91 quasars, 3 FR II galaxies, 10 BL Lac objects, 17 BL Lac/HPQs, and 6 sources without optical counterparts). In this sample 12 sources lack redshift information.

In Figure 1 we show the luminosity-redshift distribution of sample based on the data of Lister \& Homan (2005), as well as the flux density cutoffs corresponding to the northern and southern sky regions. The smallest and largest observed luminosities in our sample are $L_{\min }^{\mathrm{obs}} \approx 1.19 \times 10^{25} \mathrm{~W} \mathrm{~Hz}^{-1}$ and $L_{\max }^{\mathrm{obs}} \approx 1.03 \times$ $10^{29} \mathrm{~W} \mathrm{~Hz}^{-1}$ and the redshifts range from $z_{\min }=0.0491$ to $z_{\text {max }}=3.408$.

\subsection{Missing Redshifts}

Despite considerable observational effort, the redshift information on the MOJAVE sample is incomplete, because of the featureless optical spectra of several blazars, and weak/obscured optical counterparts. We address this problem by building a pool of redshifts from sources which have known redshifts and flux densities within $0.15 \mathrm{Jy}$ of the source with the unknown redshift. We then randomly select a redshift from this pool to be used as the redshift for that source. Alternatively, one could randomly select redshifts from the entire pool of 102 sources for known FR

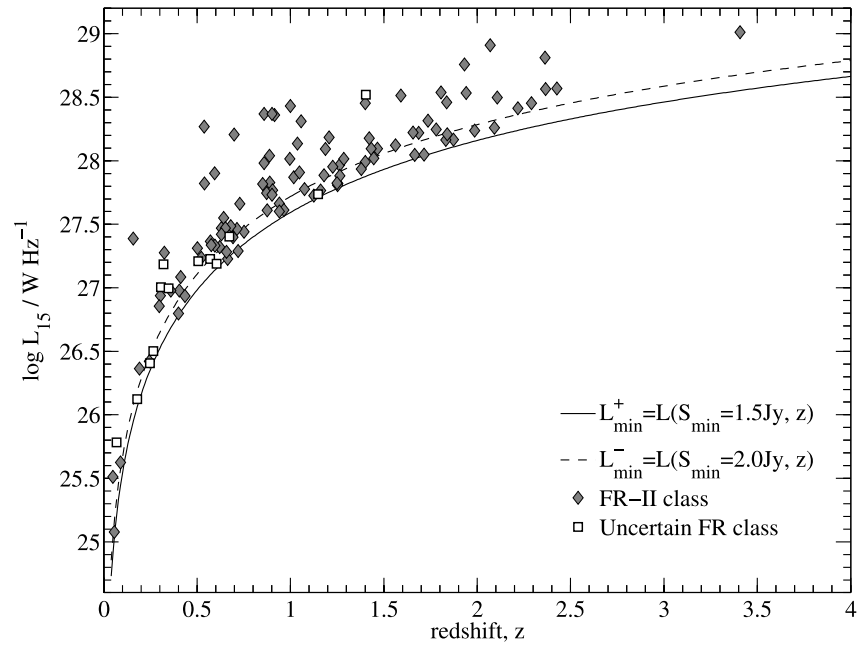

FIG. 1.-Luminosity-redshift distribution of the full MOJAVE sample (omitting 4 known FR I galaxies and two GPS sources). Only sources with known redshifts are plotted. We use diamonds for known FR II sources and open squares for sources of uncertain FR class. The solid line corresponds to the $1.5 \mathrm{Jy}$ flux density cutoff for the sources with positive J2000.0 declinations and the dashed line corresponds to the 2 Jy flux density cutoff for the sources with negative declinations, assuming a flat spectral index.

II sample (115 for the full sample); however, we chose the former method because of the large range of luminosity and redshift spanned by the sample. Because there is only one missing redshift in the known FR II sample, the number of possible redshift combinations is only 13 for the known FR II sample, compared to $4.3 \times 10^{13}$ for the full sample. In the discussion that follows, we use 13 (for the known FR II sample) and 1000 (for the full sample) realizations of the randomized redshifts to determine the statistical errors on our best-fit model parameters arising from missing redshift information.

\section{METHOD}

\subsection{Parameterized Luminosity Function}

The differential luminosity function of a population of objects is defined as the number of objects per unit comoving volume per unit luminosity interval, i.e.,

$$
\phi(\mathcal{L}, z)=\frac{d^{2} N(\mathcal{L}, z)}{d V d \mathcal{L}}
$$

where $N$ is the number of objects of luminosity $\mathcal{L}$ found in the comoving volume $V$ at redshift $z$. Studies of flux-limited AGN samples using the $\left\langle V / V_{\max }\right\rangle$ test, including MOJAVE (Arshakian et al. 2006), indicate that the RLF generally evolves with redshift. Without losing generality, we can write the RLF as

$$
\phi(\mathcal{L}, z)=\phi_{0}(\mathcal{L}) f_{\mathrm{ev}}(\mathcal{L}, z)
$$

where $\phi_{0}(\mathcal{L})$ is the local $(z \simeq 0) \operatorname{RLF}$ and $f_{\text {ev }}(\mathcal{L}, z)$ is the evolution function.

For the intrinsic RLF, we adopt a parameterization in which the local RLF is a simple power law of the form

$$
\phi(\mathcal{L})= \begin{cases}\frac{n_{0}}{L_{*}}\left(\frac{\mathcal{L}}{L_{*}}\right)^{\alpha}, & \mathcal{L}_{1}<\mathcal{L}<\mathcal{L}_{2}, \\ 0, & \text { elsewhere }\end{cases}
$$


where $L_{*}$ is an arbitrary constant with units of luminosity and $n_{0}$ is a normalization constant. In this paper we will use $L_{*}=10^{27} \mathrm{~W} \mathrm{~Hz}^{-1}$.

Traditionally, the evolution (in the simplest cases taken to be luminosity-independent) has been parameterized in two popular forms: a power-law evolution of the form $(1+z)^{k}$, or an exponential evolution of the form $\exp [k \tau(z)]$ where $\tau(z)$ is the lookback time. Other studies (e.g., Willott et al. 1998) have used one- or two-tailed Gaussian redshift dependencies. We were not able to successfully fit the MOJAVE data using these parameterizations. In particular, in several cases such parameterizations predicted a large spike in the number of low-redshift sources, which is not the case for the MOJAVE sample. Instead, we found that a good fit to the data could be obtained using the following luminosityindependent density evolution function:

$$
f_{\mathrm{ev}}(\mathcal{L}, z)=f_{D}(z) \equiv z^{m} \exp \left[-\frac{1}{2}\left(\frac{z-z_{0}}{\sigma}\right)^{2}\right],
$$

where $m, z_{0}$, and $\sigma$ are free parameters of the model. Note that this function does not reduce to $f_{D}(z)=1$ at $z=0$; we therefore assume that the model evolution function is valid for a range of redshifts $z_{1}<z<z_{2}$. Combining equations (2)-(4), our intrinsic model RLF becomes

$$
\phi(\mathcal{L}, z)=\frac{n_{0}}{L_{*}}\left(\frac{\mathcal{L}}{L_{*}}\right)^{\alpha} z^{m} \exp \left[-\frac{1}{2}\left(\frac{z-z_{0}}{\sigma}\right)^{2}\right],
$$

which is valid over the domain

$$
\mathcal{L}_{1}<\mathcal{L}<\mathcal{L}_{2}, \quad z_{1}<z<z_{2}
$$

Because the luminous jet material is moving with a speed comparable to $c$ (bulk Lorentz factor $\gamma \gg 1$ ), its observed monochromatic luminosity will be boosted as

$$
L=\delta^{p} \mathcal{L},
$$

where $\mathcal{L}$ is the luminosity in the rest frame, $p=2-\alpha_{\text {rad }}$ for continuous jet emission, $\alpha_{\text {rad }}$ is the spectral index, and $\delta$ is the kinematic Doppler factor defined as

$$
\delta=\left(\gamma-\sqrt{\gamma^{2}-1} \cos \theta\right)^{-1}
$$

where $\gamma=\left(1-\beta^{2}\right)^{-1 / 2}$ is the Lorentz factor and $\beta=v / c$ is the velocity of the emitting plasma. If the viewing angle to the jet lies within the range $0^{\circ} \leq \theta \leq 90^{\circ}$ and $\gamma_{1} \leq \gamma \leq \gamma_{2}$, then the possible Doppler factors range from

$$
\delta_{\min }=1 / \gamma_{2}
$$

to

$$
\delta_{\max }=\gamma_{2}+\sqrt{\gamma_{2}^{2}-1}
$$

If the intrinsic luminosity $\mathcal{L}$ is Doppler boosted as in equation (7), then the distribution of the observed luminosities $L$ will be different from the distribution of the intrinsic luminosities. Following the approach used by Lister (2003), we derive the form of the observed RLF of the Doppler beamed sources:

$$
\Phi(L, z)=\frac{n_{0}}{L_{*}}\left(\frac{L}{L_{*}}\right)^{\alpha} f_{D}(z) \int_{\delta_{1}(L)}^{\delta_{2}(L)} P_{\delta}(\delta) \delta^{-p(\alpha+1)} d \delta
$$

where $P(\delta)$ is the probability density function for $\delta$. This model function is valid over the domain

$$
L_{1}<L<L_{2}, \quad z_{1}<z<z_{2}
$$

where

$$
\begin{gathered}
L_{1} \equiv \delta_{\min }^{p} \mathcal{L}_{1}, \\
L_{2} \equiv \delta_{\text {max }}^{p} \mathcal{L}_{2} .
\end{gathered}
$$

In equation (11), the limits of integration $\delta_{1}(L)$ and $\delta_{2}(L)$ are given by

$$
\begin{aligned}
& \delta_{1}(L)=\min \left\{\delta_{\max }, \max \left[\delta_{\min },\left(L / \mathcal{L}_{2}\right)^{1 / p}\right]\right\}, \\
& \delta_{2}(L)=\max \left\{\delta_{\min }, \min \left[\delta_{\max },\left(L / \mathcal{L}_{1}\right)^{1 / p}\right]\right\} .
\end{aligned}
$$

where $\delta_{\min }$ and $\delta_{\max }$ are given by equations (9) and (10). The probability density function for $\delta$ is

$$
P_{\delta}(\delta)=\delta^{-2} \int_{f(\delta)}^{\gamma_{2}} \frac{P_{\gamma}(\gamma)}{\sqrt{\gamma^{2}-1}} d \gamma
$$

where $P_{\gamma}(\gamma)$ is the probability density function for $\gamma$, and the lower limit of integration is given in equation (A6) of Lister (2003). According to the previous results of Lister \& Marscher (1997) we adopt a power-law form of $P_{\gamma}(\gamma)$ with index $k$ :

$$
P_{\gamma}(\gamma)=C \gamma^{k}
$$

for $\gamma_{1}<\gamma<\gamma_{2}$, where $C$ is a normalization constant.

For computational purposes we express $P_{\delta}(\delta)$ using $\beta$-functions (see the Appendix, eq. [A9]) as

$$
\begin{aligned}
P_{\delta}(\delta)= & \frac{C}{2 \delta^{2}}\left\{B\left(1-\frac{1}{\gamma_{2}^{2}}, \frac{1}{2},-\frac{k}{2}\right)\right. \\
& \left.-B\left[1-\frac{1}{f^{2}}(\delta), \frac{1}{2},-\frac{k}{2}\right]\right\} .
\end{aligned}
$$

\subsection{Maximum Likelihood Method}

From equation (11) it is apparent that the model parameters $\left(\alpha, m, z_{0}, \sigma\right)$ of the Doppler-beamed RLF are the same as the parameters of the intrinsic RLF. Therefore, we can find the parameters of the intrinsic RLF by fitting the Doppler-beamed RLF to the observed data. For this purpose we use the maximum likelihood method of Marshall et al. (1983), which attempts to minimize the function $S=-2 \ln$ (Likelihood). The integral in $S$ (eq. [2] of Marshall et al. 1983) should be equal to the sample size $N$ for a good fit. Therefore, we must minimize

$$
S\left(\alpha, m, z_{0}, \sigma\right)=-2 \sum_{i=1}^{N} \ln \left[\Phi\left(L_{i}, z_{i}\right)\right]+2 N,
$$

and normalize $\Phi(L, z)$ such that

$$
\begin{aligned}
N= & f_{\Omega}^{+} \int_{z_{1}}^{z_{2}} d z \frac{d V}{d z} \int_{\max \left[L_{1}, L_{\min }^{+}(z)\right]}^{L_{2}} d L \Phi(L, z) \\
& +f_{\Omega}^{-} \int_{z_{1}}^{z_{2}} d z \frac{d V}{d z} \int_{\max \left[L_{1}, L_{\min }^{-}(z)\right]}^{L_{2}} d L \Phi(L, z),
\end{aligned}
$$


where $N$ is the sample size, and $f_{\Omega}^{+} \approx 6.00912 / 4 \pi$ and $f_{\Omega}^{-} \approx$ $2.08012 / 4 \pi$ are the fractional area of the sky available to the survey (in this section the plus sign superscript refers to the northern sky area while the minus sign superscript refers to the southern sky area: $0^{\circ}<\delta \leq-20^{\circ}$ ). In equation (21) we take into account that in the MOJAVE sample we have two different nonoverlapping sky areas, each with its own flux density limit: $S_{\min }^{+}=$ $1.5 \mathrm{Jy}$ and $S_{\min }^{-}=2.0 \mathrm{Jy}$. The $L_{\min }^{+}(z)$ and $L_{\min }^{-}(z)$ in the equation (21) are the monochromatic luminosity limits corresponding to the flux density limits of the survey:

$$
L_{\min }^{ \pm}(z)=4 \pi S_{\min }^{ \pm} D_{L}^{2}(z)(1+z)^{-\left(1+\alpha_{\mathrm{rad}}\right)},
$$

where $D_{L}(z)$ is the luminosity distance. To minimize $S\left(\alpha, m, z_{0}, \sigma\right)$ we use the AMOEBA algorithm from Press et al. (1992).

Other parameters of the model, such as the redshift limits $\left(z_{1}\right.$ and $\left.z_{2}\right)$, luminosity limits $\left(\mathcal{L}_{1}\right.$ and $\left.\mathcal{L}_{2}\right)$, power-law exponent $k$ of the Lorentz factor distribution (eq. [18]) and its range of possible values $\left[\gamma_{1}, \gamma_{2}\right]$ are taken as fixed a priori, and are not included in the set of optimized parameters. Some of these parameters (e.g., $\mathcal{L}_{1}$ and $k$ ) are poorly constrained (for reasons explained at the end of this section), while others can be estimated from the data directly, as follows.

The sources in both the FR II only sample as well as in the full sample span a broad range of redshifts from $z_{\min }=0.0491$ to $z_{\max }=3.408$. Later in this tion we show the upper limit of the intrinsic luminosity of the parent population to be about $\mathcal{L}_{2}=$ $10^{29} \mathrm{~W} \mathrm{~Hz}^{-1}$. The flux limit of the MOJAVE survey would allow the detection of luminous sources with $\mathcal{L} \geq 10^{28} \mathrm{~W} \mathrm{~Hz}^{-1}$ at $z=4$ if their Doppler factors are $\delta \geq 2.15$. The lack of such sources in the MOJAVE sample at redshifts $z \gtrsim 3.4$ may be of importance in modeling the RLF of the parent population. Therefore, by extending the range of redshifts to $z=4$, we allow more freedom in the optimization procedure. In addition, this will ensure that we do not exclude the statistical possibility of some objects at higher redshift. Because of this we slightly extend the redshift range and set

$$
z_{1}=0.04, \quad z_{2}=4
$$

Extending the upper redshift limit to higher values should not have a large effect on the model RLF, since the flux cutoff of the survey will make the available comoving volume very small at high $z$ (see, e.g., Willott et al. 1998). In addition, because of the above mentioned lack of sources above $z \sim 3.4$, the optimization process will constrain the evolution function (eq. [4]) to vanish rapidly at larger redshifts.

Previous studies (see, e.g., Homan et al. 2006; Cohen et al. 2007) have shown that the apparent speeds of powerful AGN jets are closely related to their bulk flow velocities and VLBI core properties. Since the MOJAVE sample contains powerful AGN with highly core-dominated radio structures (Cooper et al. 2007) and superluminal jets (Kellermann et al. 2004), one might expect the parent population to have $\gamma_{1} \gg 1$. However, the parent population likely contains sources with much lower jet speeds, and indeed, Cohen et al. (2007) estimates that the jet speed in Cygnus A (one of the sources in our subsamples) is $0.59<\beta<$ 0.68. Other authors (e.g., Wardle \& Aaron 1997) obtain similar estimates for jet speeds in kiloparsec scales outflows $(\beta \geq 0.6)$. Assuming no strong deceleration of the jets in FR II sources, we adopt the value of $\beta_{\min }=0.6$, or $\gamma_{1}=1.25$, for the minimum jet speeds in the parent population. We will discuss the effects of this choice on our model LF in $\S 4$ 4.1. Using recent observational data, Cohen et al. (2007) find that for the MOJAVE sample, $\gamma_{\max } \approx 32$. Lister \& Marscher (1997) find, using Monte Carlo simulations, that a power-law exponent of the Lorentz factor distribution in the range $-1.5 \lesssim k \lesssim-1.75$ provide a reasonable fit to the CJ-F survey (Taylor et al. 1996), a comparable radioloud blazar sample. In this paper we consider the following range of possible Lorentz factors and the exponent $k$ :

$$
\gamma_{1}=1.25, \quad \gamma_{2}=32, \quad k=-1.5
$$

We can estimate the lower and upper limits for the intrinsic luminosity as follows. First, from equations (9), (10), and (24) we obtain

$$
\delta_{\min }=1 / \gamma_{2}=0.031, \quad \delta_{\max }=\gamma_{2}+\sqrt{\gamma_{2}^{2}-1} \approx 64
$$

and we can apply the equation (7) to the observed luminosity range in our sample: $L_{\text {min }}^{\mathrm{obs}} \approx 1.19 \times 10^{25} \mathrm{~W} \mathrm{~Hz}^{-1}$ and $L_{\max }^{\mathrm{obs}} \approx$ $1.03 \times 10^{29} \mathrm{~W} \mathrm{~Hz}^{-1}$. For example, in the extreme case where the range of intrinsic luminosities $(\mathcal{L})$ is maximized, we have $L_{\text {min }}^{\text {obs }}=\mathcal{L}_{1} \delta_{\text {max }}^{2}$ and $L_{\text {max }}^{\text {obs }}=\mathcal{L}_{2} \delta_{\text {min }}^{2}$, and we obtain $\mathcal{L}_{1} \approx 3.31 \times$ $10^{21} \mathrm{~W} \mathrm{~Hz}^{-1}$ and $\mathcal{L}_{2} \approx 1.05 \times 10^{32} \mathrm{~W} \mathrm{~Hz}^{-1}$. In reality, there is a very low probability of having such extreme values in the MOJAVE sample (see, e.g., Cohen et al. 2007). To fine tune this range, we initially fit the data using the values given above. We used the parameters of the resulting fitted RLF to produce a large population of sources via Monte Carlo simulations. We examined the intrinsic luminosity distribution of a simulated fluxlimited sample to see if many sources had intrinsic luminosities near the value of $\mathcal{L}_{1}$. If all sources were well above this value, we adjusted $\mathcal{L}_{1}$ upward incrementally until we obtained a tight fit of the simulated distribution of the intrinsic luminosities to the initial range used in that particular step. A similar procedure was applied for the upper limit $\mathcal{L}_{2}$. In this manner we found that for the known FR II sample

$$
\begin{aligned}
& \mathcal{L}_{1}=10^{22.2} \approx 1.58 \times 10^{22} \mathrm{~W} \mathrm{~Hz}^{-1}, \\
& \mathcal{L}_{2}=10^{29.1} \approx 1.26 \times 10^{29} \mathrm{~W} \mathrm{~Hz}^{-1},
\end{aligned}
$$

provided a good fit to the simulated intrinsic luminosity histogram. Similarly, for the full sample we find the following limits for the intrinsic luminosities

$$
\begin{aligned}
& \mathcal{L}_{1}=10^{21.6} \approx 3.98 \times 10^{21} \mathrm{~W} \mathrm{~Hz}^{-1}, \\
& \mathcal{L}_{2}=10^{29.2} \approx 1.58 \times 10^{29} \mathrm{~W} \mathrm{~Hz}^{-1} .
\end{aligned}
$$

For these Monte Carlo simulations we have used the 64 bit random number generator (RNG) of Marsaglia \& Tsang (2004) as many commonly used algorithms (e.g., Press et al. 1992) lack the necessary resolution for generating deviates that a needed to span the wide range of luminosities found in equations (25)-(28).

Substituting the above range of intrinsic luminosities into equations (13) and (14), we obtain a theoretical range for the observed luminosities: $L_{1} \approx 1.55 \times 10^{19} \mathrm{~W} \mathrm{~Hz}^{-1}$ and $L_{2} \approx 5.15 \times$ $10^{32} \mathrm{~W} \mathrm{~Hz}^{-1}$ for the known FR II sample and $L_{1} \approx 3.89 \times$ $10^{18} \mathrm{~W} \mathrm{~Hz}^{-1}$ and $L_{2} \approx 6.49 \times 10^{32} \mathrm{~W} \mathrm{~Hz}^{-1}$ for the full sample. These ranges are much larger than the observed range of luminosities in the MOJAVE sample (see $L_{\min }^{\mathrm{obs}}$ and $L_{\max }^{\mathrm{obs}}$ above). Because the model RLF is not well determined outside the observed luminosity range, we adopt a conservative approach and adopt the following validity range for the luminosities of the observed (beamed) RLF: $L_{1}=10^{25} \mathrm{~W} \mathrm{~Hz}^{-1}$ and $L_{2}=1.1 \times 10^{29} \mathrm{~W} \mathrm{~Hz}^{-1}$. 
Using the values of $\mathcal{L}_{1}$ and $\mathcal{L}_{2}$ from equations (25) and (26), we find that the MOJAVE cutoff luminosities are too high for us to observe some important features of the RLF. For example, from Figure 3 of Lister (2003) it is evident that we would need to observe below the luminosity $L_{4} \approx 6.49 \times 10^{25} \mathrm{~W} \mathrm{~Hz}^{-1}$ for the known FR II sample $\left(L_{4} \approx 1.63 \times 10^{25} \mathrm{~W} \mathrm{~Hz}^{-1}\right.$ for full sample; see Lister 2003, eq. [9]) to probe the region of the RLF that is most susceptible to the changes in values of the lower luminosity $\mathcal{L}_{1}$ of the parent population and power-law index $k$. But in our sample we have too few sources with $L<L_{4}$. For these reasons we chose to estimate some parameters of the model from the data as described above, and not to include them in the set of optimized parameters.

\section{RESULTS}

\subsection{Model Parameters}

Using our adopted form of density evolution (eq. [4]) and parameters from equations (23), (24), (25), and (26), we minimized the quantity $S\left(\alpha, m, z_{0}, \sigma\right)$ for 1000 (182 for the known FR II sample) randomizations of missing redshifts as described in $\S 2.2$. For each fitted parameter, we took the median of the distribution as the best-fit value of the respective parameter. The best-fit values of the model RLF thus obtained are presented in Table 2. The error estimates for model parameters have been obtained using the $\Delta S=1$ method (see, Lampton et al. 1976). (For a given parameter we maximized the likelihood function while keeping all other parameters constant. We then varied this parameter until a variation $\Delta S=1$ was obtained.) We have also obtained an estimation of the error in the parameter due to missing redshift information at the level of $1 \sigma$ from the values of the parameters for which the fractional cumulative distribution function was equal to either 0.683 or 0.317 . We found that these errors are negligible compared to the errors computed using the $\Delta S=1$ method. We calculated the normalization constant $n_{0}$, space density $\rho$ for $L>1.3 \times 10^{25}$, and parent population $K$ using the best-fit values for the model parameters $\alpha, m, z_{0}$, and $\sigma$ so that equation (20) yielded the sample size $N=103$ for the known FR II sample and $N=127$ for the full sample. The errors on $n_{0}$ and $K$ were also calculated using their cumulative distribution functions as described above. We have evaluated the goodness of fit of our model RLFs using the two-dimensional KolmogorovSmirnov (K-S) test as described in Press et al. (1992). The Kolmogorov-Smirnov probability $\left(P_{\mathrm{KS}}\right)$ is a $p$-value that shows the probability of observing a K-S test statistic $\left(D_{\mathrm{KS}}\right)$ as large or larger than observed one and can be used to reject a model if its value is too small. We will accept a model if $P_{\mathrm{KS}} \geq 0.2$. The K-S probabilities for our models indicated good fits to the data with Monte Carlo realizations of missing redshifts, ranging from 0.67 to 0.79 for the known FR II sample (with $D_{\mathrm{KS}}$ from 0.067 to 0.079 ) and from 0.40 to 0.97 for the full sample (with $D_{\mathrm{KS}}$ from 0.048 to 0.089 ).

From Table 2 we can see that while the slope for the intrinsic luminosity distribution of the FR II only sample is slightly shallower than the slope of the full sample, the parameters of the density evolution functions of the two samples agree to within $1 \sigma$, suggesting that the objects of uncertain FR class (9 BL Lac objects, 9 BL Lac/HPQ sources, and 6 sources without optical classification) may actually be of the FR II class.

The median of average space densities (for $L>1.3 \times$ $10^{25} \mathrm{~W} \mathrm{~Hz}^{-1}$ ) computed for 13 (1000 for the full sample) randomizations of the unknown redshifts is $\approx 1580 \mathrm{Gpc}^{-3}$ for the known FR II sample and $\approx 4390 \mathrm{Gpc}^{-3}$ for the full sample. For the full sample the space density is quite large. We speculate that
TABLE 2

Best-fit Model Luminosity Function Parameters

\begin{tabular}{|c|c|c|}
\hline Parameter & FR II only & All except FR I \\
\hline$\alpha \ldots \ldots$ & $-2.53 \pm 0.06$ & $-2.65 \pm 0.06$ \\
\hline$m$ & $1.4 \pm 0.1$ & $1.6 \pm 0.1$ \\
\hline$z_{0}$ & $1.29 \pm 0.09$ & $1.18 \pm 0.09$ \\
\hline$\sigma$ & $0.76 \pm 0.09$ & $0.8 \pm 0.1$ \\
\hline$n_{0}\left(\mathrm{Mpc}^{-3}\right) \ldots \ldots \ldots \ldots$ & $(2.87 \pm 0.04) \times 10^{-10}$ & $(2.22 \pm 0.04) \times 10^{-10}$ \\
\hline$\rho\left(\mathrm{Gpc}^{-3}\right) \ldots \ldots \ldots \ldots \ldots$ & $(1.579 \pm 0.008) \times 10^{3}$ & $(4.39 \pm 0.07) \times 10^{3}$ \\
\hline 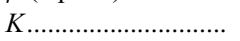 & $(5.49 \pm 0.04) \times 10^{9}$ & $(1.55 \pm 0.03) \times 10^{11}$ \\
\hline $\mathcal{L}_{1}\left(\mathrm{~W} \mathrm{~Hz}^{-1}\right) \ldots \ldots \ldots$ & $10^{22.2}$ & $10^{21.6}$ \\
\hline $\mathcal{L}_{2}\left(\mathrm{~W} \mathrm{~Hz} \mathrm{~Hz}^{-1}\right) \ldots \ldots \ldots$ & $10^{29.1}$ & $10^{29.2}$ \\
\hline
\end{tabular}

Notes.-The errors in the parameters $\alpha, m, z_{0}$, and $\sigma$ of the model LF were computed using the $\Delta S=1$ method as described in the text. The error estimates on the normalization factor $n_{0}$, space density $\rho$ for $L>1.3 \times 10^{25} \mathrm{~W} \mathrm{~Hz}^{-1}$ and parent population $K$ were calculated using their cumulative distribution functions as described in $\S 4.1$.

this may indicate that the RLF has a different slope for lower luminosities, but the lack of low luminosity sources in our sample does not allow us to verify this hypothesis. We can also explain this increase in the space density for the full sample by an underestimation of the lower intrinsic luminosity $\mathcal{L}_{1}$ (see eq. [27]) due to the large number of sources with missing redshifts in the full sample.

As discussed in $\S 3.2$, the value of the lower limit of the Lorentz factors $\gamma_{1}$ is not very well constrained, with some authors (e.g., Arshakian et al. 2006) suggesting higher values (e.g., $\gamma_{1}=3$ ) than the one adopted here $\left(\gamma_{1}=1.25\right)$. Therefore, to investigate whether or not the choice of a particular value of $\gamma_{1}$ has a significant influence on the model RLF, we have repeated the computations for the FR II only sample using $\gamma_{1}=3$. While we have obtained a slightly different intrinsic luminosity range $\left(\mathcal{L}_{1}=\right.$ $10^{21.8} \mathrm{~W} \mathrm{~Hz}^{-1}$ and $\mathcal{L}_{2}=10^{29} \mathrm{~W} \mathrm{~Hz}^{-1}$ ), the model RLF parameters are essentially unchanged: $\alpha=-2.55 \pm 0.06, m=$ $1.48 \pm 0.14, z_{0}=1.27 \pm 0.09$, and $\sigma=0.76 \pm 0.10$. This relative independence of the results on a particular choice of the lower limit $\gamma_{1}$ for the Lorentz factors is due to the insensitivity of the bright end $\left(L>L_{4}\right)$ of the beamed RLF to the values of $\gamma_{1}$ and $\gamma_{2}$ (see Lister 2003 Fig. 5). However, the fact that the luminosity functions in Figure 5 of Lister (2003) differ strongly at lower luminosities than $L_{4}$ means that the choice of $\gamma_{1}$ potentially could modify the predicted parent population sizes. Indeed, for the FR II only sample with $\gamma_{1}=3$, we obtain $\mathrm{K}=(1.7 \pm$ $0.1) \times 10^{10}$, which is about 3 times larger than the parent population predicted by the RLF computed with $\gamma_{1}=1.25$, but this could be due to the lower value of $\mathcal{L}_{1}$ obtained when $\gamma_{1}=3$.

In Figure 2 we present the integral source counts $N(>S)$ per unit of solid angle for the observed data (known FR II sample), and as predicted by our fitted RLF after it is beamed. The $1 \sigma$ error bars in this and subsequent figures are computed according to Poisson statistics using the method of Gehrels (1986).

\subsection{Redshift Distribution}

In Figure 3 we plot the binned redshift distribution and the associated $1 \sigma$ error bars for the known FR II sample (with the missing redshifts replaced with the averages of the "redshift pools" as described in $\S 2.2$ ). The solid line represents the predicted redshift distribution for our best-fit model, while the faint gray lines show the distributions for the 13 randomizations of the missing redshifts. We can see that while the missing redshift information creates a tangible uncertainty in the redshift distribution, we obtain a reasonably good overall fit to the data. 


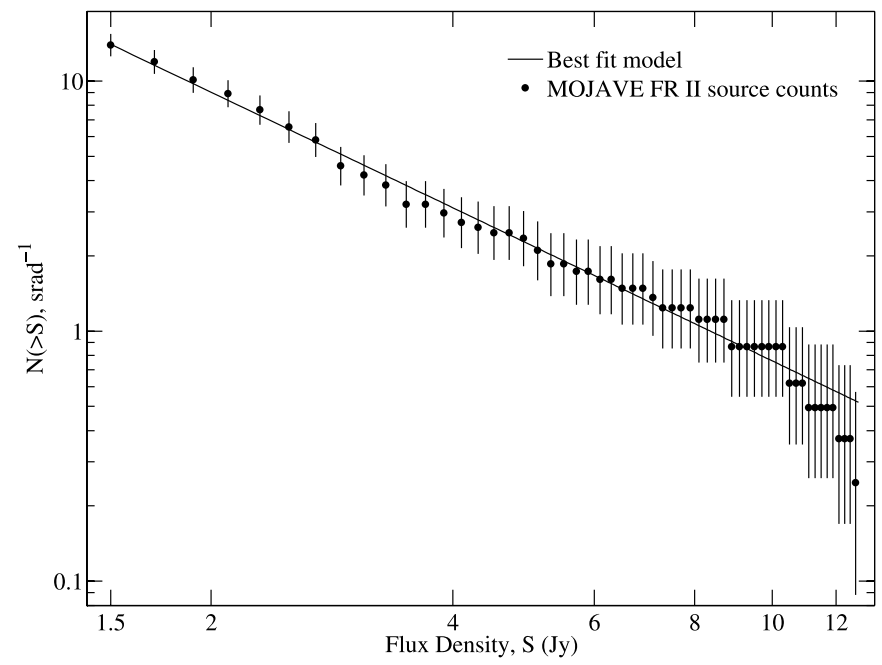

FIG. 2. - Integral source count of the known FR II sample $N(>S)$ per unit of solid angle.

\subsection{Radio Luminosity Function}

We use the method of Page \& Carrera (2000) to construct the observed luminosity function. In this method, we compute the value of the RLF in a bin with a luminosity interval $L_{\min }$ and $L_{\max }$ and a redshift interval $z_{\min }$ and $z_{\max }$ :

$$
\Phi_{\mathrm{est}}=\frac{N}{\int_{z_{\min }}^{z_{\max }} \int_{L_{\min }(z)}^{L_{\max }}(d V / d z) d z d L},
$$

and its uncertainty:

$$
\delta \Phi_{\mathrm{est}}=\frac{\delta N}{\int_{z_{\min }}^{z_{\max }} \int_{L_{\min }(z)}^{L_{\max }}(d V / d z) d z d L},
$$

where $N$ is the number of objects in the bin, $\delta N$ its uncertainty, and $L_{\min }(z)$ is the minimum luminosity within the bin at which we can still detect an object. In equations (29) and (30) we have switched the order of integration compared to the original formulation of Page \& Carrera (2000).

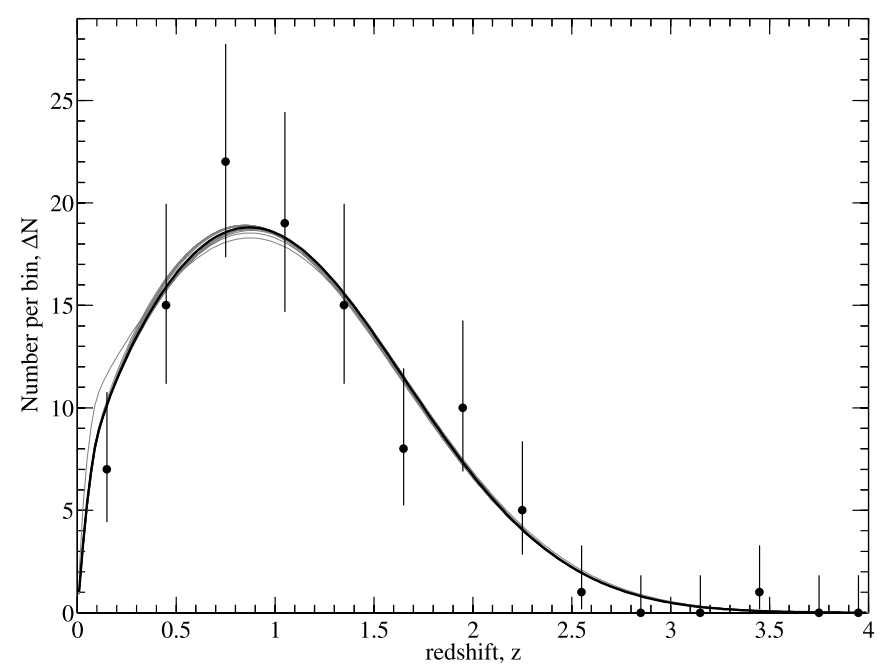

FIG. 3.- Plot of model redshift distributions for each redshift randomization (thin light gray lines), best-fit model distribution (thick black line), and the observed redshift distribution of the MOJAVE known FR II sample ( filled circles with error bars corresponding to $1 \sigma$ confidence level).

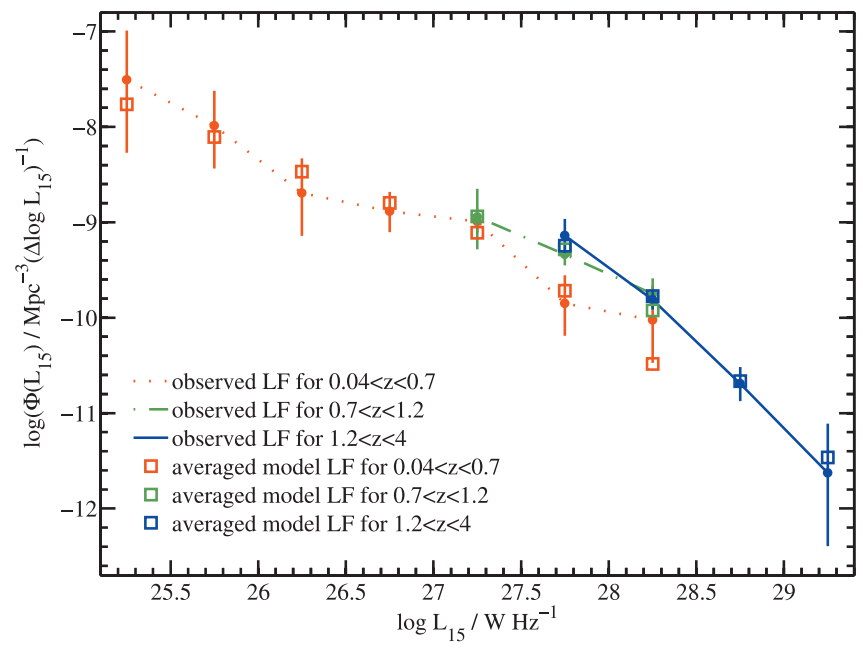

FIG. 4.- Observed binned RLF of the MOJAVE known FR II sample ( filled circles and lines) and the binned model RLF (open squares) for three redshift intervals: $0.04<z<0.7$ (red, dotted line), $0.7<z<1.2$ (green, dotdashed line), and $1.2<z<4$ (blue, solid line). The luminosity bin width is $\Delta \log L=0.5$.

We use these same equations to compute the binned (i.e., averaged over a luminosity-redshift bin) model RLF. We believe this is the most robust way to compare the observed and model RLF when binning is involved. We use equations (21) to compute the effective number of sources $N$ in the luminosity-redshift bin of interest (i.e., we replace $z_{1}, z_{2}, L_{1}$, and $L_{2}$ in eq. [21] with $z_{\min }, z_{\min }, L_{\min }$, and $L_{\max }$ of the luminosity-redshift bin of interest).

The fitted and observed RLFs for the MOJAVE sample are presented in the Figure 4. It is apparent that the averaged model RLF provides a good fit to the sample data. At first glance, it would also appear that both the fitted and observed RLFs obey a broken power law that steepens at higher luminosities. While a beamed LF can have different slopes for different luminosity intervals (see e.g., Urry \& Shafer 1984; Lister 2003), this is does not explain the observed break in the observed (beamed) LF (e.g., because $L_{4}$ is too low for our sample; see Fig. 5). Based on this Arshakian et al. (2006) claimed that a double power-law intrinsic

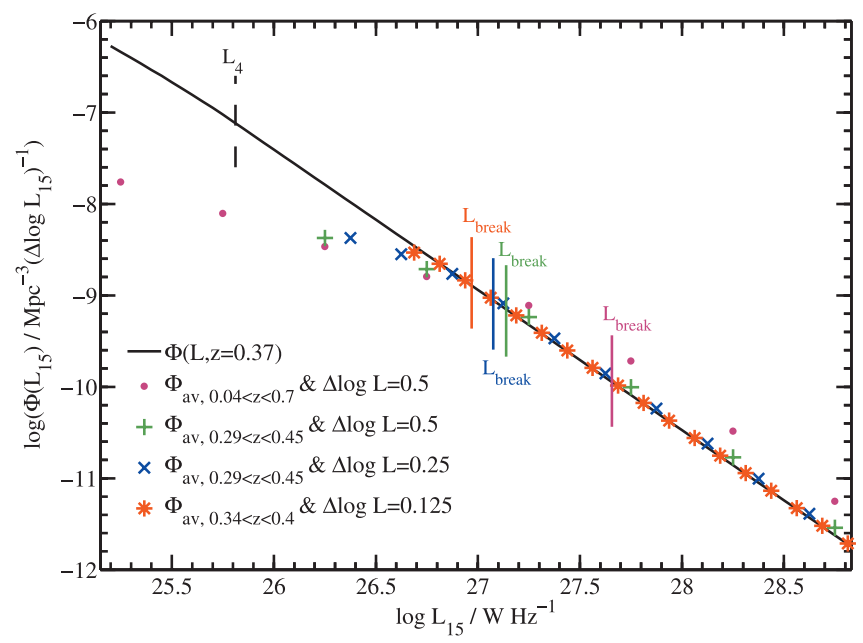

FIG. 5.-Differential model LF $\Phi(L, z)$ and differential LFs averaged over bins of varying size. The best agreement between the differential LF and its binned version is obtained for smaller bin sizes. For large bin sizes the binned (or averaged over bins) LF flattens for lower luminosities. The vertical lines show the "break" luminosities for each LF: $\log L_{\text {break }}=\log L_{\min }^{-}\left(S_{\min }^{-}=2 \mathrm{Jy}, z_{\max }^{\text {bin }}\right)+(\Delta \log L) / 2$. 


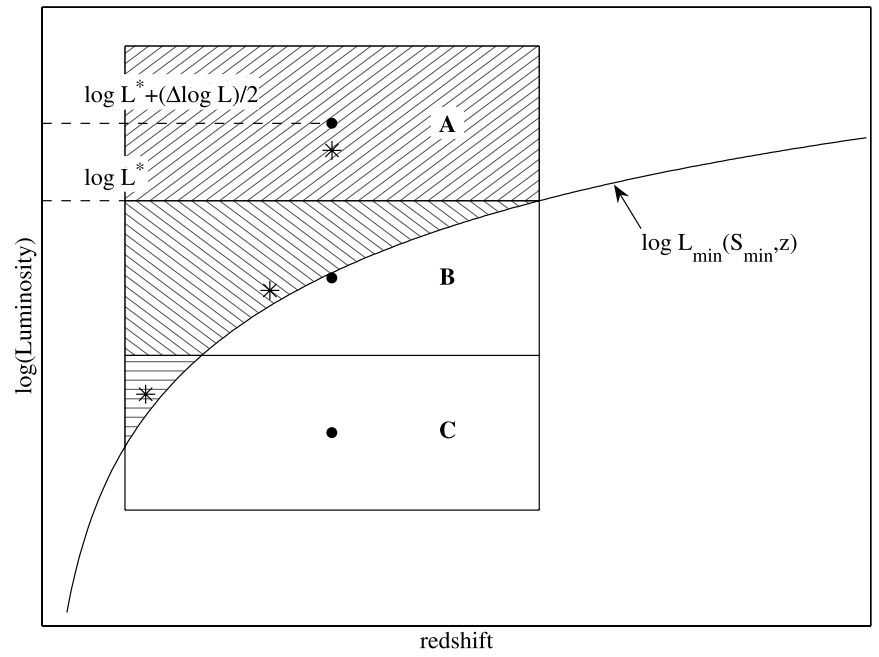

FIG. 6.- Illustration of distortions induced by binning and flux cutoff on the binned LF. The curved line indicates the flux cutoff: $L_{\min }\left(S_{\min }, z\right)$. Rectangles represent bins in the $\log$ (Luminosity)-redshift plane. The value of the binned LF is computed as the average of the differential LF over hatched regions of the bins. Filled circles represent the position of the center of the bins (the luminosity coordinates of these centers were used to plot the binned LF in Fig. 4 and in Fig. 5). The asterisks show the position of the "center of mass" of the hatched regions for the bins assuming a $\Phi(L, z)=L^{-2.5}$. Because of the power-law distribution of luminosities $\left(L^{-2.5}\right)$, the center of mass for the bin " $\mathrm{A}$ " lies below its geometrical center. If, in addition, evolution is present, then the center of mass will shift along the redshift axis as well.

LF is needed to describe the observed (beamed) LF. However, we find that this is an artifact of the binning method.

In Figure 5 we plot the Doppler beamed differential model RLF for the known FR II sample as well as its average over bins of varying sizes. We can see that our differential Doppler beamed RLF is in fact very close to a simple power-law RLF, with only a slight flattening for $L<L_{4}$. Because of the large bins, the agreement between the binned RLF (Fig. 4), and the differential RLF (Fig. 5) is apparent only at high luminosities. This can be improved by using smaller bin sizes, but at the expense of larger Poisson errors. For a steep RLF with strong evolution across a bin, large bins can create apparent breaks in the observed RLFs when these bins intersect the luminosity cutoff of the sample (see eq. [22]). This is because the averages of RLFs computed over parts of the bins above the luminosity cutoff will be very different from the averages computed over whole bins (see Fig. 6). A superior way to plot a binned LF would be to use centers of mass of the bins over which averaging is done instead of their geometrical centers but unfortunately, this is almost impossible to accomplish without a priori knowledge of the luminosity function slope and evolution parameters. We can see from Figure 6 that the center of mass of the bins is different from the geometrical center, even for bins lying entirely above the flux cutoff (i.e., bin " $A$ " in Fig. 6). The presence of a redshift dependence in the luminosity function will shift the center of mass of the bins along the redshift axis as well. The chopped bins will not be centered around the same redshifts as the whole bins, and therefore, the average of the RLF computed over a chopped bin should actually belong to a RLF computed at a different cosmological epoch. That is, by assuming that the value of the RLF at the center of the bin is equal to the average RLF computed over a smaller part of the bin (e.g., the hatched area of the bin " $C$ " in Figure 6, which may even not contain the center of the bin) one can introduce a large error in the case of strongly evolving functions. In Figure 7 we present a zoomed in version of the Figure 5 on which, additionally, we plot the "break" luminosities. We can

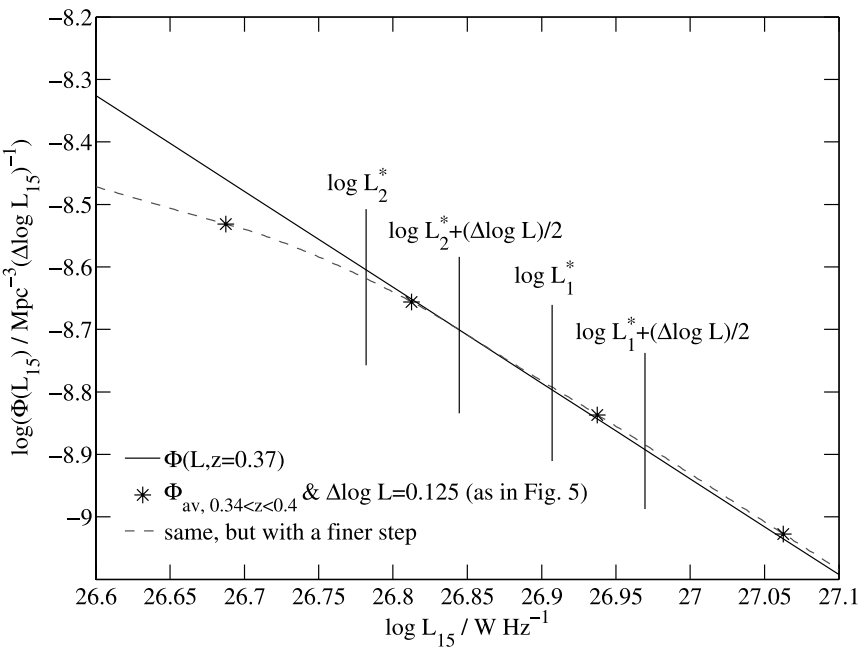

FIG. 7.-Differential model LF $\Phi(L, z)$ (continuous line) and the differential LF averaged over bins of size $\Delta z=0.06(0.34<z<0.4)$ and $\Delta \log L=0.125$ (the binned LF represented by red asterisks in Fig. 5; dashed line and asterisks). Asterisks are placed at the same positions as in Fig. 5. The vertical lines show the "break" luminosities $\log L_{1}^{*}+(\Delta \log L) / 2$ and $\log L_{2}^{*}+(\Delta \log L) / 2$ where $L_{1}^{*}=L_{\min }^{+}\left(S_{\min }^{+}=1.5 \mathrm{Jy}, z=0.4\right)$ and $L_{2}^{*}=L_{\min }^{-}\left(S_{\min }^{-}=2 \mathrm{Jy}, z=0.4\right)$.

see that the shifting of the center of mass toward lower luminosities for full bins produce a shift of the LF to the right for $\log L>\log L_{2}^{*}+(\Delta \log L) / 2$. For $\log L<\log L_{2}^{*}+(\Delta \log L) / 2$, the LF flattens because of the shifting of the center of mass for the chopped bins toward larger luminosities as well as toward smaller redshifts (see Fig. 6). We conclude that the double power law that we see in the observed RLF is, therefore, an artifact created by the effect of flux density cutoff on steep power law of the intrinsic RLF combined with a strong evolution of the luminosity function across a bin. This double power law is not a property of the intrinsic RLF of the MOJAVE sample as Arshakian et al. (2006) concluded. In fact, we are able to obtain good fit using a simple power-law intrinsic RLF.

As previously mentioned in $\S 3.2$, the RLF depends most strongly on the lower luminosity cutoff $\mathcal{L}_{1}$ and Lorentz factor distribution power-law index $k$ at luminosities smaller than $L_{4}$. At larger luminosities it appears more like a featureless simple power law, as illustrated in Figure 5. Indeed, from mathematical considerations, the slope of the beamed RLF is expected to be nearly identical to the slope of the unbeamed (intrinsic) RLF for luminosities between $L_{4} \approx 6.49 \times 10^{25} \mathrm{~W} \mathrm{~Hz}^{-1}$ and $L_{8} \approx 5 \times$ $10^{29} \mathrm{~W} \mathrm{~Hz}^{-1}$ for the known FR II sample and $L_{4} \approx 1.63 \times$ $10^{25} \mathrm{~W} \mathrm{~Hz}^{-1}$ and $L_{8} \approx 4.8 \times 10^{29} \mathrm{~W} \mathrm{~Hz}^{-1}$ (see Lister 2003; Urry et al. 1991).

\section{CONCLUSIONS}

We have analyzed the redshift and flux density distributions of a complete sample of AGN selected on the basis of relativistically beamed $15 \mathrm{GHz}$ radio flux density (MOJAVE) to derive the parent luminosity function of bright radio-loud blazars. We carried out our analysis on two samples, one consisting of only the 103 known FR II class radio sources in MOJAVE ("the known FR II sample") and a "full sample" that added 24 sources of uncertain FR class.

1. We find that the observed MOJAVE RLF can be well-fit using a Doppler-boosted, single power law intrinsic RLF with slope $\alpha=-2.53 \pm 0.06$ for the known FR II sample ( $\alpha=$ $-2.65 \pm 0.06$ for the full sample), and a density evolution function of the form $z^{m} \exp \left\{-1 / 2\left[\left(z-z_{0}\right) / \sigma\right]^{2}\right\}$, with parameters 
$m=1.4 \pm 0.1, z_{0}=1.29 \pm 0.09$, and $\sigma=0.76 \pm 0.09$ for the known FR II sample ( $m=1.6 \pm 0.1, z_{0}=1.18 \pm 0.09$, and $\sigma=$ $0.80 \pm 0.1$ for the full sample). We assumed a power-law Lorentz factor distribution with the exponent $k=-1.5$ and $1.25<\gamma<$ 32. Our model is valid over the range $0.04<z<4$ in redshift, $10^{22.2} \mathrm{~W} \mathrm{~Hz}^{-1}<\mathcal{L}<10^{29.1} \mathrm{~W} \mathrm{~Hz}^{-1}$ in intrinsic luminosity for the known FR II sample $\left(10^{21.6} \mathrm{~W} \mathrm{~Hz}^{-1}<\mathcal{L}<10^{29.2} \mathrm{~W} \mathrm{~Hz}^{-1}\right.$ for the full sample), and $10^{25} \mathrm{~W} \mathrm{~Hz}^{-1}<L<1.1 \times 10^{29} \mathrm{~W} \mathrm{~Hz}^{-1}$ in observed luminosity.

2. We find a good agreement between the fitted RLF parameters of the two samples, suggesting that the objects of uncertain FR class in the MOJAVE sample (9 BL Lac objects, 9 intermediate quasar/BL Lac sources, and 6 sources without optical classification) may in fact belong to the FR II class.

3. We have shown that the double power-law shape of the observed (i.e., beamed) MOJAVE RLF is an artifact due to large changes of the evolving RLF across a bin and its interaction with the lower luminosity cutoff of the survey. We find no evidence for a break in the intrinsic blazar RLF above $10^{25} \mathrm{~W} \mathrm{~Hz}^{-1}$ at $15 \mathrm{GHz}$.
The authors wish to acknowledge the contributions of the other members of the MOJAVE project team: Hugh and Margo Aller, Tigran Arshakian, Marshall Cohen, Dan Homan, Matthias Kadler, Ken Kellermann, Yuri Kovalev, Andrei Lobanov, Eduardo Ros, Rene Vermeulen, and Tony Zensus. We also thank the anonymous referee for helpful comments and suggestions that improved this paper.

M. Cara wishes to thank Andrzej Lewicki and Michael Sloothaak for providing additional computational resources at Purdue University.

This research was supported by NSF grant 0406923-AST, a grant from the Purdue Research Foundation, and made use of the following resources: the NASA/IPAC Extragalactic Database (NED), which is operated by the Jet Propulsion Laboratory, California Institute of Technology, under contract with the National Aeronautics and Space Administration, and the University of Michigan Radio Astronomy Observatory, which is supported by the National Science Foundation and by funds from the University of Michigan.

\section{APPENDIX}

\section{REPRESENTATION OF $P_{\delta}(\delta)$ USING INCOMPLETE $\beta$-FUNCTIONS}

In order to avoid direct numerical integration, it is convenient to express the $P_{\delta}(\delta)$ through the $\beta$-functions and then use the continuedfraction representation (see Press et al. 1992) for fast computation of the integral.

If

$$
P_{\gamma}(\gamma)=C \gamma^{k}
$$

then

$$
P_{\delta}(\delta)=\delta^{-2} \int_{f(\delta)}^{\gamma_{2}} \frac{P_{\gamma}(\gamma)}{\sqrt{\gamma^{2}-1}} d \gamma=C \delta^{-2} \int_{f(\delta)}^{\gamma_{2}} \frac{\gamma^{k}}{\sqrt{\gamma^{2}-1}} d \gamma \equiv C \delta^{-2} G\left(k, f(\delta), \gamma_{2}\right)
$$

where we have defined

$$
G\left(k, z_{1}, z_{2}\right) \equiv \int_{z_{1}}^{z_{2}} \frac{\gamma^{k}}{\sqrt{\gamma^{2}-1}} d \gamma
$$

Making the substitution $t \equiv \gamma^{2}$, this integral can be rewritten:where

$$
B(z, a, b) \equiv \int_{0}^{z} t^{a-1}(t-1)^{b-1} d t
$$

is the incomplete $\beta$-function defined for $0 \leq z \leq 1, a>0$, and $b>0$. In our problem $z_{2}>z_{1} \geq 1$, and therefore we represent the $\beta$-function through the hypergeometric function

$$
B(z, a, b)=a^{-1} z^{a}{ }_{2} F_{1}(a, 1-b, a+1 ; z),
$$

and continue it analytically into the region $|z|>1$ using the formula (see Landau \& Lifshitz 1989, their eq. [6])

$$
\begin{aligned}
{ }_{2} F_{1}(\alpha, \beta, \gamma ; z)= & \frac{\Gamma(\gamma) \Gamma(\beta-\alpha)}{\Gamma(\beta) \Gamma(\gamma-\alpha)}(-z)^{-\alpha}{ }_{2} F_{1}\left(\alpha, \alpha+1-\gamma, \alpha+1-\beta ; \frac{1}{z}\right) \\
& +\frac{\Gamma(\gamma) \Gamma(\alpha-\beta)}{\Gamma(\alpha) \Gamma(\gamma-\beta)}(-z)^{-\beta}{ }_{2} F_{1}\left(\beta, \beta+1-\gamma, \beta+1-\alpha ; \frac{1}{z}\right), \text { for }|z|>1
\end{aligned}
$$


We then obtain

$$
\begin{aligned}
B(z, a, b)= & a^{-1} z^{a}{ }_{2} F_{1}(a, 1-b, a+1 ; z) \\
= & a^{-1} z^{a}\left[\frac{\Gamma(a+1) \Gamma(1-a-b)}{\Gamma(1-b) \Gamma(1)}(-z)^{-a}{ }_{2} F_{1}\left(a, 0, a+b ; \frac{1}{z}\right)\right. \\
& \left.+\frac{\Gamma(a+1) \Gamma(a+b-1)}{\Gamma(a) \Gamma(a+b)}(-z)^{b-1}{ }_{2} F_{1}\left(1-b, 1-a-b, 2-a-b ; \frac{1}{z}\right)\right], \\
= & \Gamma(a+1) a\left[(-1)^{-a} \frac{\Gamma(1-a-b)}{\Gamma(1-b)}\right. \\
& \left.+(-1)^{b-1} \frac{\Gamma(a+b-1)}{\Gamma(a) \Gamma(a+b)}(1 z)^{1-a-b}{ }_{2} F_{1}\left(1-a-b, 1-b, 2-a-b ; \frac{1}{z}\right)\right], \\
= & (-1)^{-a} \frac{\Gamma(a+1) \Gamma(1-a-b)}{a \Gamma(1-b)}+(-1)^{b} B\left(\frac{1}{z}, 1-a-b, b\right),
\end{aligned}
$$

and therefore

$$
G\left(k, z_{1}, z_{2}\right)=-\frac{1}{2}\left[B\left(\frac{1}{z_{2}^{2}},-\frac{k}{2}, \frac{1}{2}\right)-B\left(\frac{1}{z_{1}^{2}},-\frac{k}{2}, \frac{1}{2}\right)\right], k \neq 2 m(m=0,1,2, \ldots),
$$

which can be computed using a continued fraction method (see Press et al. 1992). For situations when $k$ is close to $2 m(m=0,1,2, \ldots)$ we can use the relationship

$$
B(z, a, b)=B(a, b)-B(1-z, b, a)
$$

to get

$$
G\left(k, z_{1}, z_{2}\right)=\frac{1}{2}\left[B\left(1-\frac{1}{z_{2}^{2}}, \frac{1}{2},-\frac{k}{2}\right)-B\left(1-\frac{1}{z_{1}^{2}}, \frac{1}{2},-\frac{k}{2}\right)\right] .
$$

Finally,

$$
P_{\delta}(\delta)=C 2 \delta^{2}\left[B\left(1-\frac{1}{\gamma_{2}^{2}}, \frac{1}{2},-\frac{k}{2}\right)-B\left(1-\frac{1}{f^{2}}(\delta), \frac{1}{2},-\frac{k}{2}\right)\right] .
$$

\section{REFERENCES}

Antonucci, R. 1993, ARA\&A, 31, 473

Arshakian, T. G., Ros, E., \& Zensus, J. A. 2006, A\&A, 458, 397

Cassaro, P., Stanghellini, C., Bondi, M., Dallacasa, D., della Ceca, R., \& Zappalà, R. A. 1999, A\&AS, 139, 601

Cohen, M. H., Lister, M. L., Homan, M., Kadler, D. C., Kellermann, K. I.,

Kovalev, Y. Y., \& Vermeulen, R. C. 2007, ApJ, 658, 232

Cooper, N. J., Lister, M. L., \& Kochanczyk, M. D. 2007, ApJS, 171, 376

Gehrels, N. 1986, ApJ, 303, 336

Homan, D. C., et al. 2006, ApJ, 642, L115

Jackson, C. A., \& Wall, J. V. 1999, MNRAS, 304, 160

Kellermann, K. I., et al. 2004, ApJ, 609, 539

Kotilainen, J. K., Hyvänen, T., \& Falomo, R. 2005, A\&A, 440, 831

Kovalev, Y. Y., et al. 2005, AJ, 130, 2473

Lampton, M., Margon, B., \& Bowyer, S. 1976, ApJ, 208, 177

Landau, L. D., \& Lifshitz, E. M. 1989, Quantum Mechanics: Non-relativistic

Theory, Vol. 3 (4th ed.; Moscow: Nauka Publishing)

Ledlow, M. J., \& Owen, F. N. 1996, AJ, 112, 9

Lister, M. L. 2001, ApJ, 561, 676

$$
\text { 2003, ApJ, 599, } 105
$$

Lister, M. L., \& Homan, D. C. 2005, AJ, 130, 1389

Lister, M. L., \& Marscher, A. P. 1997, ApJ, 476, 572

1999, Astropart. Phys., 11, 65

Marsaglia, G., \& Tsang, W. W. 2004, Statistics Probab. Lett., 66, 183
Marshall, H. L., Avni, Y., Tananbaum, H., \& Zamorani, G. 1983, ApJ, 269, 35 Owen, F. N., \& Ledlow, M. J. 1994, in ASP Conf. Ser. 54, The First Stromlo Symposium: The Physics of Active Galaxies, ed. G. V. Bicknell, M. A. Dopita, \& P. J. Quinn (San Francisco: ASP), 319

Padovani, P., \& Urry, C. M. 1992, ApJ, 387, 449

Pagani, C., Falomo, R., \& Treves, A. 2003, ApJ, 596, 830

Page, M. J., \& Carrera, F. J. 2000, MNRAS, 311, 433

Press, W. H., Teukolsky, S. A., Vetterling, W. T., \& Flannery, B. P. 1992, Numerical Recipes: The Art Of Scientific Computing (Cambridge: Cambridge Univ. Press)

Rector, T. A., \& Stocke, J. T. 2001, AJ, 122, 565

Taylor, G. B., Vermeulen, R. C., Readhead, A. C. S., Pearson, T. J., Henstock, D. R., \& Wilkinson, P. N. 1996, ApJS, 107, 37

Urry, C. M., \& Padovani, P. 1991, ApJ, 371, 60 1995, PASP, 107, 803

Urry, C. M., Padovani, P., \& Stickel, M. 1991, ApJ, 382, 501

Urry, C. M., \& Shafer, R. A. 1984, ApJ, 280, 569

Vermeulen, R. C., \& Cohen, M. H. 1994, ApJ, 430, 467

Veron-Cetty, M.-P., \& Veron, P. 2000, Astron. Astrophys. Rev., 10, 81 2006, A\&A, 455, 773

Wardle, J. F. C., \& Aaron, S. E. 1997, MNRAS, 286, 425

Willott, C. J., Rawlings, S., Blundell, K. M., \& Lacy, M. 1998, MNRAS, 300, 625 